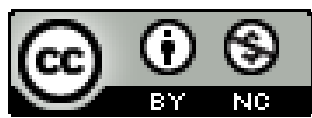

\title{
LA MÁQUINA DE ESCRIBIR DE HEIDEGGER: LOS OBJETOS TÉCNICOS COMO EJEMPLOS EN SU FILOSOFÍA
}

\author{
Heidegger's typewriter: technological objects as examples in his \\ philosophy
}

\section{Luciano Mascaro CONICET/UBA}

\begin{abstract}
Resumen: Este trabajo se propone responder una pregunta: ¿hasta qué punto se puede utilizar la filosofía de Heidegger para discutir temáticas de filosofía de la tecnología?. Por medio de contrastaciones de los dichos y ejemplos propuestos por Heidegger con los resultados de los estudios de diversas ciencias empíricas, la investigación mostrará una serie de limitaciones en el rango de aplicación de las afirmaciones, específicamente, del Heidegger posterior a los años "30. Por esta vía buscamos explicitar un hecho conocido pero no muy frecuentemente mencionado: las afirmaciones del segundo Heidegger acerca de la técnica, se realizan con independencia de investigaciones empíricas y estudios de casos, puesto que su interés es ontológico y no historiográfico o arqueológico. Esto representa una limitación si se intenta utilizar su filosofía para estudiar cuestiones asociadas a la estructura de los objetos técnicos concretos. El objetivo final de este trabajo es demarcar las posibilidades y las restricciones de esta filosofía si se busca aplicarla al mundo tecnificado y al estudio de la estructura y diseño de los objetos que lo pueblan.
\end{abstract}

Palabras clave: Heidegger, ejemplo, filosofía de la tecnología, artefacto

\begin{abstract}
This article intends to answer one question: up to which point can Heidegger's philosophy be utilized to discuss themes of philosophy of technology? Through the testing of Heidegger's notions and examples against the results of various empirical sciences, this research will show a series of limitations in the application scope of heideggerian statements, specifically after the 1930 's. By doing this, we seek to make explicit a known, but not frequently mentioned situation: Heidegger's statements about technology after the 1930 's are formulated with independence from empirical researches and case-studies, because his interest is ontologicall, not historiographical or archeological. This constitutes a limitation, if his philosophy is to be used in the study of issues asociated with the structure of concrete technological objects. The main objective of this study is to demarcate the possibilities and restrictions of this phillosophy, if an attempt to apply it to the technified world and the structure and design of the objects that populate it is to be made.
\end{abstract}

Keywords: Heidegger, example, philosophy of technology, artifact.

\section{Introducción}

Dentro del área temática conocida como filosofía de la tecnología, frecuentemente se menciona a Heidegger como uno de los representantes del tratamiento filosófico de la estructura de los objetos técnicos. Esta mención suele ir acompañada de una referencia a los análisis heideggerianos de útilles, tales como puentes, jarras, zapatos, molinos y martillos. Ahora bien, ¿es el análisis heideggeriano de casos, en verdad un aporte al estudio filosófico de los útiles? ¿o acaso dichos análisis cumplen con alguna otra función?. Si se analizan los ejemplos de objetos técnicos y su tematización correlativa en los textos heideggerianos posteriores a los años ' 30 , rápidamente se podrán encontrar imprecisiones en lo referente a las descripciones de 
sus funcionamientos, orígenes históricos, experiencias de uso, estructuras materiales y causales operantes en ellos, y acerca de los efectos y consecuencias de su involucramiento en un entramado social, simbólico y cultural. Con esta situación en mente, la estrategia de este escrito se organiza en torno a un fingimiento: simularemos encarnar una postura que intenta encontrar inconsistencias en el pensamiento de Heidegger acerca de la técnica por medio de la contrastación de sus afirmaciones con los datos obtenidos de investigaciones científicas positivas. Una y otra vez, encontraremos que nuestra búsqueda de inconsistencias resulta infructuosa puesto que para desarrollar esta tarea, se deben interpretar los casos mencionados por Heidegger como ejemplos en sentido tradicional, empírico e inductivo, y llevar dichos casos al terreno de la observación científica; no solo una tarea que a la que Heidegger no se abocó, sino también un modo de interpretación que el propio autor desaconseja ${ }^{1}$. Por medio de este infructuoso fingimiento, perseguimos un objetivo puntual: hacer manifiesto hasta qué punto Heidegger (específicamente, después de la Kehre de los años '30) no es en verdad. un pensador de la estructura y funcionamiento de los objetos técnicos.

El hecho de que Heidegger no permita una lectura empírica e inductiva de sus ejemplos y que no haya intentado un estudio empírico ingenieril de objetos técnicos, no nos impide a nosotros perseguir las consecuencias de ensayar una interpretación de este tipo. Intentaremos lo que un estudioso de Heidegger podría considerar una "objeción fácil", que no capta la filosofía heideggeriana en su verdadera estatura. A pesar de esta advertencia, nos ocuparemos de someter a prueba algunas afirmaciones heideggerianas acerca de objetos técnicos, para ver qué es lo que aquella advertencia heideggeriana esconde en verdad. El resultado será la indicación de cuál es el rango de acción y relevancia temática de la filosofía de la técnica del segundo Heidegger.

Para realizar esta tarea nos concentraremos en un caso particular: los dichos de Heidegger acerca de la máquina de escribir y el acto de escribir; a continuación atenderemos a algunos contraejemplos, algunos estudios históricos y científicos, y a las interpretaciones de otros filósofos que se ocuparon de analizar la técnica en general, y la máquina de escribir en particular desde una perspectiva más asentada en la experiencia de utilización y en la adquisición de habilidades de empleo.

\section{1. la mano y la escritura}

En su lección de 1943, titulada "Parménides", Heidegger realiza la siguiente afirmación: "La máquina de escribir arranca la escritura del dominio esencial de la mano, es decir, del dominio de la palabra”2. No es difícil detectar que la afirmación de Heidegger es fenomenológica e históricamente arbitraria.

En primer lugar, sometamos la afirmación de Heidegger a una contrastación (cuyo interés crítico, como ya indicamos, está destinado fracasar) con algunas nociones provenientes del ámbito de la paleontología y antropología: en la lección de 1943, Heidegger afirma que la mano es el terreno originario de la palabra y que la palabra antecede a la mano ${ }^{3}$. En contraste con esta afirmación encontramos investigaciones paleontológicas que ubican el antecedente más manifiesto de la mano humana en los fósiles del australopithecus afarensis, que vivió hace 3 millones de años. Por su parte, estudios recientes han sugerido que la aparición del lenguaje articulado en humanos primitivos puede haber ocurrido al fundirse la capacidad melódica y pragmática, evento

${ }^{1}$ Cf. M. Heidegger, Ser y Tiempo, Trotta, Madrid, 2006, p.68, y M. Heidegger, "La pregunta por la técnica", en Conferencias y artículos, ed. Del Serbal, Barcelona, p.6. La primera indicación se refiere a la interpretación que considera al Dasein como ejemplo de un género universal de entes; Heidegger desaconseja la lectura de su ejemplo como el caso particular de un universal, es decir, el camino inductivo -tradicionalmente asociado a la comprensión de los ejemplos- que va de lo singular a lo general, queda clausurado. En la segunda referencia, Heidegger indica que una constatación histórica (científica) de la relación entre física y técnica sería infructuosa para los objetivos de su estudio.

${ }^{2}$ M. Heidegger, Parmenides, Madrid, Akal, 2005, p.105

${ }^{3} \mathrm{M}$. Heidegger, Ibid.

${ }^{4}$ H.M. Schmidt y U. Lanz, Surgical Anatomy of the Hand, New York, Thieme, 2003, p 1-3. 
que debió acontecer hace unos 100.000 años ${ }^{5}$ Como puede verse, a pesar de lo que afirme Heidegger, es la mano la que antecede evolutivamente al lenguaje. Ahora bien, ¿tiene acaso valor esta contrastación con la paleontología? ¿cumple la función de indicar la imprecisión de la afirmación de Heidegger? Sin dudas, un estudioso de este autor respondería a nuestra indicación diciendo: "esto no es lo que Heidegger intenta afirmar", se nos dirá inmediatamente: "la «mano" no se refiere, en este contexto, al apéndice del cuerpo humano sino a una estructura formal, una Handlung, un modo de desocultamiento pragmático de los entes y el mundo, un modo de relación con el ser del cual solo la existencia humana es capaz, es decir, el interés de Heidegger no es histórico sino ontológico"6. Esta primera confrontación nos muestra que, al expresarse de aquel modo, Heidegger no se mueve en el ámbito de la discusión paleontológica, puesto que si lo hiciese, se encontraría en contradicción con algunos datos abiertamente aceptados por la comunidad científica. Para que su filosofía no se vea afectada por esta afirmación empíricamente imprecisa, es necesario que dicha filosofía recorra otros caminos, o que funcione con afirmaciones de un carácter diferente al empírico. El propio Heidegger es consciente de esta situación, y por ello realiza las advertencias que mencionamos.

En efecto, es evidente que Heidegger no desarrolla un planteamiento paleontológico. Lo que no es evidente -la noción que aquí quiero explicitar progresivamente- es que la imprecisión empírica, y la contrastación con estudios de caso que hablan en contra de sus afirmaciones, no afectan su filosofía. Continuemos analizando las afirmaciones heideggerianas acerca de la escritura, la mano y la máquina de escribir: Esta "historia" de los tipos de escritura es una de las principales razones para la creciente destrucción de la palabra?.

¿Cuál es esa "historia" de los tipos de escritura? Manifiestamente, Heidegger se refiere al paso del uso de pluma, al uso de estilográfica, al uso de bolígrafo, al uso de máquina de escribir. Sin embargo, como vimos hace un momento, cada vez que Heidegger realice un análisis lindante con disciplinas empíricas, debemos estar prevenidos: él no es un historiador, y su interés no se dirige a lo pasado y lo presente en términos historiográficos, sino en el sentido de la antecedencia o derivación ontológica. De alhí nuestra sospecha ante su "historia" de los tipos de escritura; y esto sin mencionar, por ejemplo, que la historia de la escritura en occidente difiere en gran medida de la escritura en oriente ${ }^{8}$.

Realicemos una segunda confrontación de los dichos de Heidegger, esta vez, con los datos obtenidos de investigaciones historiográficas, a fin de determinar si las afirmaciones del filósofo alemán se sostienen empíricamente: Según Heidegger, la historia de los tipos de escritura coincide con la progresiva "destrucción de la palabra". Nos preguntamos inmediatamente: si esto es así, ¿en qué momento se encontró la palabra "intacta", o sea, en estado "puro" u originario? Si esa historia es historia de la "degradación", podemos inferir que la forma más "pura" de la palabra se encontraba al comienzo de este devenir. Y si, según los dichos de Heidegger, resulta que la escritura pertenece al ámbito originario del uso directo de las manos, diremos que no hemos escrito de forma originaria o auténtica desde los tiempos en que realizábamos pinturas

\footnotetext{
5 S. Miyagawa et al.., "The emergence of hierarchical structure in human language", en Frontiers in psychology, 4, 71, 2013. El problema del origen del lenguaje articulado en humanos, y la variedad de teorías y aproximaciones a esta cuestión es inagotable. Sólo menciono este estudio para ofrecer un rango temporal general para la aparición dell lenguaje, desde un punto de vista paleontológico. Con todo, ninguna de las teorías actualmente en discusión afirmaría que el lenguaje articulado apareció antes de la división filogenética del Homo (hace entre 2.3 y 2.4 millones de años) del género Pan (hace entre 5 a 6 millones de años). Existe un consenso general acerca de que los Australopithecus carecían de comunicación vocal más sofisticada que la de los grandes simios (Cf. Arcadi,

${ }^{6}$ He aquí un ejemplo de un contraargumento de este tipo: “La verdad descubierta no es la de los significados concretos que tenía [el zapato] para van Gogh (o para la campesina) sino su estructura formal." A.Bertorello, "La polémica en torno a la estética ontológica de Heidegger: Schapiro, Schaeffer y Derrida", en Contrastes, revista internacional de filosofía, Ed. Universidad de Málaga: España, 2006, p.73

${ }^{7}$ M. Heidegger, Parménides, Madrid, Akal, 2005, p.105

${ }^{8}$ D. Boorstin, Los Descubridores, trad. Ed. Grijalbo: Barcelona 1986, p190 y ss.
} 
en las cavernas, hace 40.000 años $^{9}$. Los primeros sistemas de escritura articulada y no "directamente representacional" aparecen en Mesopotamia hacia el $4000 \mathrm{AC}{ }^{10}$, y fueron realizados por medio de la impresión de un estilete o Stylos sobre tablillas de madera o arcilla.

Como podemos ver, las primeras manifestaciones de escritura humana aparecen, desde el comienzo asociadas a medios de impresión, en este caso, el estilete. En esta experiencia de escritura cuneiforme, ya puede observarse una cierta mediación entre la mano y la inscripción de la palabra, una postergación de la mano, un alejamiento entre el decir y el escribir. Esto significa, si seguimos a Heidegger, que cuando la escritura nace, su relación con la palabra ya habría sido destruida. Lo cual es absurdo.

¿En verdad se verifica lo que Heidegger supone? ¿puede haber, o ha habido alguna vez una relación propia o auténtica de la mano con la escritura? ¿Es posible una relación con la escritura independiente del empleo de un artefacto? Desde luego, es posible escribir con los dedos, pero esa práctica también implica haber conquistado uno de los artificios más complejos y pluridimensionales de nuestra cultura: el alfabeto. El mismo Heidegger incluye a la expresión lingưística articulada dentro de su análisis de los objetos técnicos ${ }^{11}$. Esa forma más auténtica del escribir, a la que apunta la nostalgia de Heidegger no se verifica históricamente. Tendremos que aceptar que nunca hemos escrito, ni podremos jamás escribir sin que una herramienta "arrebate la palabra" del supuesto ámbito originario de la mano; la escritura es tecnología.

Si tomamos esta postura, veremos que resulta completamente arbitrario escoger un punto en el cual comienza la degradación de la palabra. La escritura cuneiforme ya habría degradado la palabra por intervención de un artefacto, o más precisamente dos: el estilete, y la tablilla de arcilla (tres, si mencionamos el alfabeto). No existe algo así como una "escritura auténtica". Tal vez esta expresión, este anhelo, provenga de la predilección de Heidegger por sistemas de vida más simples, artesanales. Ahora bien, una vez más, nuestro contraejemplo histórico se encontrará, seguramente con la respuesta de un estudioso de Heidegger, quien nos diría: "Heidegger no intenta realizar un análisis histórico; la «palabra» y los «tipos de escritura» no refieren a las expresiones materiales impresas en tablillas o en papiros, sino a estructuras formales; la «palabra» es el espacio de acontecimiento de la verdad del ser, la cual sólo en sentido derivativo se expresa en signos gráficos, fijados por la presión de un útil sobre una superficie maleable". Encontramos aquí un segundo callejón sin salida. Los dichos de Heidegger tampoco pueden ser contrastados con datos obtenidos de investigaciones historiográficas, puesto que incurriríamos en una incomprensión y una falta de fidelidad a los intereses del pensador. Pero volvamos a la problemática de la escritura: Heidegger nos indica que: La escritura maquinal despoja a la mano de su rango en el dominio de la palabra escrita y degrada la palabra a un medio de comunicación ${ }^{12}$.

La cuestión de la mano como el dominio esencial ya ha sido puesta en duda (aunque infructuosamente) al considerar que la escritura, desde sus inicios ha tenido un aspecto artefactual o maquinal, es decir, la mano nunca ha escrito sin intermediarios técnicos.

Con seguridad, como ya indicamos, la afirmación de Heidegger recién expuesta intenta indicar lo siguiente: "sólo un ente que existe en el modo de relación significativa con el mundo es capaz de entablar una conexión de empleo y utilización manual; la mano, equiparada con la palabra nos demuestra que el uso manual ocupacional o la manufactura de obras de arte, $\mathrm{y}$ de acciones humanas "corporizadas" en general pertenece al horizonte que sólo se abre para el ser humano: el horizonte del sentido (como palabra) y significatividad" Con todo, Heidegger necesita utilizar ejemplos históricos para ilustrar sus indicaciones y se refiere a objetos culturales concretos. Y es

\footnotetext{
${ }^{9}$ M. Aubert, "Palaeolithic cave art in Borneo".en Nature 564, p. 254-257.

${ }^{10}$ Adkins, L. Empires of the plain, New York, St. Martin's press, 2003, p.47

${ }^{11}$ M. Heidegger, Ser y Tiempo, Madrid, Trotta, 2006, p.103 y ss. De estas afirmaciones surgirá el tratamiento de Robert Brandom del enunciado como un artefacto.

${ }_{12}$ M. Heidegger, Parmenides, Madrid, Akall, 2005, p.105 
allí, en la imprecisión de los ejemplos donde se revela la verdadera dirección ${ }^{13}$ de sus análisis. Lo que mueve al autor alemán es un diagnóstico, realizado de antemano que podría expresarse así: "la técnica moderna tiene el potencial de destruirnos, por medio de la nivelación de todos los modos de descubrimiento del ente al modo calculador y provocador". El recelo de Heidegger ante la técnica lo lleva a realizar afirmaciones con una muy endeble base empírica como la que ahora estudiamos, a saber, que la mecanización de la escritura provoca la destrucción de la palabra. Concentrémonos ahora específicamente, en el artefacto conflictivo: la máquina de escribir. ¿Es este artefacto ocasión para la completa objetivación del mundo? ¿es esta máquina funcional a la Gestell? Analicemos una serie de posturas alternativas.

\title{
2. La máquina de escribir y la Gestell
}

En una entrevista realizada en 1996 para la revista "la quincena literaria", Jacques Derrida se refiere a la cuestión de la máquina de escribir, específicamente, opina acerca de la afirmación heideggeriana que anunciaba la degradación de la palabra por medio de la mecanización. El autor francés comenta:

\begin{abstract}
Heidegger recuerda que el trabajo del pensamiento es uin trabajo de mano, una Handlung, una «acción», ante cualquier oposición entre práctica y teoría. El pensamiento sería en ese sentido una Handlung, vina maniobra, una «manera», incluso una manipulación. ¿Pero es esto una razón para protestar contra la máquina? Con la máquina de escribir o el ordenador no se prescinde de la mano sino que entra en función otra mano, otro mando -si se me permite decirlo asíotra inducción, otra orden del cuerpo a la mano y de la mano a la escritura ${ }^{14}$.
\end{abstract}

Como puede verse, para Derrida, la introducción de la máquina de escribir no degrada, sino que sólo modifica la relación de la mano con la palabra. De hecho, según nos dice, en la máquina de escribir, son los 5 dedos de las dos manos los que se avocan a la tarea de la escritura. Según él, no es la mano la que marca la distinción entre una pluma y una máquina de escribir, puesto que la mano se encuentra presente en ambas. La diferencia es el tipo de relación entre mano, artefacto y tarea, ninguna de las cuales rebaja la operación humana, al contrario, una de ellas, la más moderna, implica el ejercicio de muchos más movimientos finos, y el uso de las dos manos en lugar de una. Es falso que la máquina de escribir funcione como impedimento, obstáculo o filtro, y si lo hiciese, lo haría en el mismo sentido en que lo hace el lápiz.

En la conferencia de 1945 Heidegger afirma:

Además, la máquina de escribir ofrece la "ventaja" de ocultar el manuscrito y con ello, el carácter. En la máquina de escribir, todos los hombres tienen el mismo aspecto $^{15}$.

Según el fragmento, la palabra mecanografiada equipararía todas las escrituras, y por ello borraría el esencial rasgo personal del trazo. En contraste, Derrida indica que la relación personalizante que se da entre la escritura, la mano y el autor del escrito, tan valorada por Heidegger, sólo ha llegado a ser apreciada a finales de la edad media. Con anterioridad, la transcripción de textos era una labor anónima, en la que la relación del manuscrito con el amanuense resultaba irrelevante.

\footnotetext{
${ }^{13}$ No la contradicción, puesto que sus afirmaciones no son contradictorias, dentro de su propio sistema filosófico.

${ }^{14} \mathrm{~J}$. Derrida, "El tratamiento del texto", Entrevista con Béatrice y Louis Seguin, en La quinzaine littéraire, 698, 1996 pp. 4-7, traducción de R. Ibáñez y M. J. Pozo en No escribo sin luz artificial, cuatro ediciones, Valladolid, p. 4

${ }_{15}$ M. Heidegger, Parmenides, Madrid, Akal, 2005, p.105 
Hasta una época relativamente reciente, que podemos situar a finales de la Edad Media, la transcripción que conservamos, el texto, no es nunca la del autor, la de su puiño y letra. Con el manuscrito autógrafo aparece una nueva configuración que durará varios siglos y de la que ahora nos estamos apartando ${ }^{16}$.

Esto nos muestra que la historia de la relación entre la mano y el carácter individual del productor es una invención reciente, y no se extrae necesariamente del uso de instrumentos manuales de escritura. La fijjación del carácter personal de la "letra" sólo se vuelve relevante a partir del renacimiento, con la "invención del sujeto"17 y el surgimiento de la categoría de "autor". Lo que para Heidegger caracteriza ontológicamente, originariamente y desde siempre al uso de instrumentos manuales de escritura, en realidad es una práctica que sólo fue llevada a primer plano hace cuatro siglos, y que hoy vuelve a disolverse con la nueva estandarización de los tipos de letra y los procesadores de texto. No es esencial a la escritura el fijar la personalidad en el trazo del autor ${ }^{18}$, de hecho, la escritura manual ha sido indiferente al escritor durante mucho más tiempo que el que ha sido solidaria con esta función. Una vez más, las afirmaciones de Heidegger no se verifican históricamente. Según nos indica Derrida, ni siquiera los griegos, reverenciados por Heidegger, se interesaban por la inscripción de la individualidad en la letra ${ }^{19}$. Con todo, ante esta confrontación histórica (esta vez, a cargo de Derrida) podría volver a esgrimirse el argumento del defensor de Heidegger, a saber, que el interés del autor no es historiográfico, sino ontológico, y que la palabra y la escritura a la que nos referimos aquí constituyen en verdad la condición de posibilidad de la relación del ser humano con el mundo y la verdad del ser. La filosofía de Heidegger permanece ilesa, puesto que él no despliega un estudio de tipo historiográfico. Nuestro contraejemplo falla una vez más. Revisemos alhora otra afirmación de Heidegger desde una perspectiva nueva.

\section{La palabra y la presión mecanizada}

Heidegger indica en su lección de 1943: [La palabra] ya no va y viene por la mano que escribe, la mano que actúa propiamente, sino por la presión mecanizada de la mano 20

¿Acaso en el uso de una máquina de escribir, la palabra no "va y viene" por la mano? Pareciera que sí, pero se trataría de una mano que actuaría impropiamente. Dejemos de lado por alhora la cuestión de si es siquiera posible pensar un modo propio o auténtico de escritura manual. Concentrémonos en la segunda parte del fragmento: la palabra ahora viene por "la presión mecanizada de la mano", y esto la degrada. Pero, ¿No es también el uso de un bolígrafo una "presión mecanizada de la mano"? ¿Cuál es la diferencia entre el uso de cada tecnología, desde un punto de vista corporal y experiencial?. Someteremos, como penúltimo intento, la indicación de Heidegger a una contrastación, no ya de tịpo paleontológico o historiográfico, sino más bien fenomenológico (centrado en captación subjetiva de la experiencia del escribir).

Para Heidegger el empleo de las teclas de la máquina de escribir constituye una especie de obstácullo, una postergación, una mediación poco fiel del acto fijar la palabra en el papel, pero manifiestamente, esta no es la experiencia del que utiliza una máquina de escribir (o diríamos hoy, el teclado de una notebook). Quien emplea una de estas

\footnotetext{
${ }^{16}$ J. Derrida, Op. Cit. , p.7.

${ }^{17}$ Con respecto a esta cuestión, recordamos la famosa frase de Foucault "el hombre es un invento reciente" (Foucault, 2008, p.17). En este mismo sentido podemos hacer mención de las propuestas de Ricoeur acerca del autor como una función interna al texto. (Ricoeur, 2006, p.103)

${ }^{18}$ A esto se le suma la ingenuidad de considerar que en la letra del texto podremos encontrar la subjetividad del autor. Esta postura, en la que resuena la naciente hermenéutica de Schleiermacher, quien buscaba "comprender al autor más que él mismo", ha sido sometida a crítica, entre otros por Paul Ricoeur.

19 “En la Grecia de los siglos V y IV en tiempos de Platón, no se valoraba el autógrafo, que hasta mucho más tarde no se empezó a idolatrar" (Derrida, 1996, p.7)

${ }^{20} \mathrm{M}$. Heidegger, Op. Cit, p.105. 
máquinas no siente su discurso ni el fluir de su pensamiento postergado, o arrebatado por la "presión mecánica" de las teclas; quien escribe habilidosamente con teclas se encuentra inmerso en el acto de escribir. El teclado se vuelve "transparente" y la operación lo atraviesa en dirección a las palabras que van quedando fijadas.

Una operación exitosa y habitual del teclado no atiende a la "presión mecanizada". Del mismo modo en el que un uso exitoso y habitual del bolígrafo no se concentra en la presión mecanizada de la diminuta esfera sobre el papel, o de los dedos sobre la punta del instrumento; o bien, del mismo modo en que un uso exitoso del martillo no presta atención al "impacto mecanizado" contra el clavo, sino al progresivo incrustarse del clavo en la madera. Así como el estilete, y más tarde, la pluma y el bolígrafo mecanizan la aplicación directa del dedo a la superficie de escritura (si este modo de inscripción se verifica siquiera históricamente), del mismo modo, previamente, la escritura ha mecanizado el habla, la cual ya había sido previamente mecanizada por el alfabeto.

En Ser y Tiempo (el verdadero aporte de Heidegger a la fillosofía de la tecnología $a^{21}$ ) se nos indica que un artefacto que posibilita el trato ocupacional se caracteriza por su no-llamatividad. La ocupación no se percata de este útil, sino que lo traspasa en dirección a la tarea que se busca realizar. Si acaso el útil se resistiese a la utilización (por ejemplo, por su avería), el mismo se mostraría ante el usuario como una cosa ocupacional que está-ahí, un ente fronterizo entre el útil y el objeto, denominado cosa usual o útil-cosa [Zeugding], este tipo de experiencias son llamadas "formas deficientes de la ocupación" 22 . Si volvemos al fragmento de Heidegger observaremos que la descripción del empleo de la máquina de escribir se concentra en "la presión mecanizada", esto significa que el empleo no ha logrado atravesar exitosamente el artefacto en dirección a la tarea, y ha debido concentrarse en el "obstaculizante" movimiento de las teclas. Tal como indica Don Ihde, el autor alemán no describe la experiencia de un usuario habilidoso, sino la de un principiante ${ }^{23}$. Heidegger toma una experiencia inicial de empleo obstaculizado como si ella se tratase del único y total modo de empleo de la máquina. Thde avanza incluso más en este sentido y sugiere que Heidegger nunca aprendió a utilizar la máquina de escribirir ${ }^{24}$

Lo importante de estas inconsistencias es lo siguiente: Heidegger olvida estudiar el proceso de adquisición de habilidades técnicas, esto es, desde un punto de vista fenomenológico, los procesos de incorporación ${ }^{25}$. Él olvida que nadie ostenta la habilidad de utilizar una herramienta desde el inicio, sino que siempre existe un proceso de adaptación, por mínimo que sea, aunque éste haya sido olvidado. Sobre esta cuestión podemos escuchar el fundamental estudio de Merleau-ponty acerca de la adquisición de habitualidades:

Cuando la dactilógraf́a ejecuta sobre el teclado los movimientos necesarios, estos moviimientos están dirigidos por uina intención, pero esta ỉntención no pro-pone las teclas del teclado como ubicaciones objetivas. Cierto es que el sujeto que aprende a dactilografiar integra, al pie de la letra, el espacio del teclado a su espacio corporal ${ }^{26}$

Merleau-Ponty indica que la adquisición de habitualidades es un modo de la comprensión; se trata de la captación de una significación, pero la "captación motriz de una significación motriz"27. Este proceso, o más precisamente, el olvido de este proceso, sumado al recelo o aversión a priori ante los objetos tecnológicos no-artesanales es en

\footnotetext{
${ }^{21}$ Esta también es la postura de Walther Zimmerli, Bemhardt Irrgang, y de Don Thde, entre otros.

${ }^{22}$ Cf. M. Heidegger, Ser y Tiempo, Trotta, Madrid, 2006, p.100.

${ }^{23}$ D. Thde, Heidegger's technologies: Postphenomenological perspectives. New York, Fordham University Press, 2010, p.124

${ }^{24}$ Los datos son tomados por Thde de Elizabieta Ettinger, y de Raymond Geuss.

${ }^{25}$ D. Thde, Op. Cit., p.79

${ }^{26}$ M. Merleau Ponty, Fenomenología de la percepción, Ed. Planeta Agostini, Barcelona: España, 1994, p.162

${ }^{27}$ M. Merleau Ponty, Ibid., p.160 
parte lo que lleva a Heidegger a declarar a la máquina de escribir como un instrumento degradante de la actividad humana.

Heidegger olvida que, en su inicio, todo manejo de herramientas se muestra como una de sus "formas deficientes de la ocupación". Si el bolígrafo es tratado como una tecnología respetuosa de la mano y la palabra, y la máquina de escribir como una herramienta degradante, es porque el autor alemán nunca conoció la experiencia de aquel que consiguió habituarse al uso de las teclas y volverse habilidoso en el empleo, al punto que su escritura llegara a "fluir" a través de las teclas. La adquisición de la habitualidad es descripta por Merleau-ponty como "un saber que está en las manos" Este saber asociado corporalmente al hacer y producir es reconocido por el primer Heidegger, y es tematizado por medio de su noción de "comprensión antepredicativa", "comprensión primaria", o simplemente "téchne", ahora bien, desde su formulación, esta noción no incluyó un estudio de los procesos de adquisición. El sujeto analizado por Heidegger es un adulto en un taller que ya ha incorporado el manejo de sus artefactos. Desde allí se elaboran las propuestas acerca de la técnica, una técnica ya adquirida, jamás aprendida, jamás sometida al ensayo y error propios de la falta de habilidad. Al análisis heideggeriano le falta la fase genética de los comportamientos técnicos. Llegado el momento de aplicar su noción de comprensión a artefactos modernos, se vuelve evidente que el proceso de adquisición nunca fue tematizado.

Don Ihde, por su parte, nos muestra una parcialización adicional presente en la teoría de Heiegger: este último nunca investigó posibilidades de la relación con artefactos que no sean las del inmediato empleo manual, relaciones que Ihde denomina "de incorporación" 29 . Otras modalidades existen, como las por él denominadas relaciones "de alteridad"30, este es el caso de un trompo al que una vez puesto en funcionamiento, simplemente se lo deja realizar su actividad, un objeto ante el cual el sujeto se retira para contentarse con la observación del proceso ${ }^{31}$. Además de este tipo también existen otras formas de relación como las relaciones hermenéuticas, relaciones de trasfondo, o de constitución mutua ${ }^{32}$.

El tradicionalismo, temor, recelo, o aversión de Heidegger es el de los hombres de edad avanzada que reniegan de la nueva tecnología por la incomodidad que les causaría tener que incorporar esquemas operacionales nuevos. ¿qué pasaría si esta adquisición se tornase demasiado dificultosa para ellos? ¿reconocerían su incapacidad y el anacronismo de sus manejos, o condenarían a la máquina, junto con las nuevas prácticas que ella acarrea como instrumento de la degradación del ser humano?

¿Aceptaría nuestro imaginario interlocutor esta indicación de la falta de tematización de la adquisición de habitualidades como un argumento capaz de desarticular un aspecto del estudio heideggeriano? ¿aceptaría que el modelo del usuario habilidoso parcializa la experiencia que se intenta estudiar?. Con seguridad, y con razón, nuevamente se nos indicaría que "la presión mecanizada de las teclas" no se refiere en verdad al ejercicio percutor de las teclas físicas de una máquina, sino al modo provocador y emplazante que aparta al ser humano del pensar; si la presión mecanizada menciona una modalidad de esa estructura formal y epocal llamada maquinación (machenschaft) entonces tampoco aquí podremos alojar una crítica empírica al pensamiento heideggeriano.

\section{El martillo multiplicado}

\footnotetext{
${ }^{28}$ M. Merleau Ponty, Ibid., p.161

${ }^{29}$ D. Thde, Op. Cit.., p.78

${ }^{30}$ D. Thde, Ibid., p.79

${ }^{31}$ Thde intuye que probablemente la relación de los usuarios contemporáneos con sus pantallas de celulares y computadoras tenga algo que ver con este tipo de relación de alteridad, donde el empleo del artefacto, 0 la interfase, tenga algo de contemplación recreativa, donde el objeto técnico se presenta como un "quasi otro". ${ }^{32}$ D. Ihde, Technics and praxis, Dordrecht: Holland, Ed. Reidel Publishing Co. 1979, cap. 1 
Deseo realizar una última contrastación, esta vez tomaré un objeto técnico analizado por Heidegger, y colocaré ese objeto en otro contexto, a fin de mostrar como un simple cambio de disposición de un artefacto es suficiente para que el mismo pase de ser tratado como un ente axiológicamente neutro, a un ente manifiestamente degradante. Tal vez el ejemplo más consagrado en el tratamiento heideggeriano de las herramientas sea el caso del martillo. Este ejemplo emerge una y otra vez en los estudios acerca de la técnica en el primer Heidegger, se trata, prácticamente, del estandarte de la filosofía de la técnica heideggeriana de los años '20. Ahora bien, en esa época, aún nada se ha dicho acerca de la Gestell, de la provocación de la naturaleza, del peligro de la homogeneización de todos los modos del desocultamiento, ni de la serenidad (Gelassenheit). Al mismo tiempo, el martillo casi no aparece en las reflexiones posteriores a los '30. Pareciera que el martillo ha escapado al sistema de alarmas heideggeriano. Sin embargo nos preguntamos: ¿no consiste la máquina de escribir, instrumento de la degradación de la palabra, precisamente en unos 45 pequeños martillos colocados en un semicírculo y accionados por la presión de los dedos, en combinación con una serie de palancas?, ¿en qué momento la combinación y articulación de instrumentos inocuos se vuelve peligrosa y degradante? El momento de conversión de lo inofensivo en amenazante es, una vez más, elegido antojadizamente; y esto puede aplicarse a gran parte de la filosofía de la técnica del segundo Heidegger.

Soy consciente de que las menciones acerca de la máquina de escribir y el estudio del martillo pertenecen a períodos diferentes del pensamiento de Heidegger; con todo, esta contrastación busca señalar dos situaciones: 1) que el verdadero estudio fenomenológico de artefactos concretos, es decir, aquel estudio que toma al artefacto como un caso empírico que es abierto fenomenológicamente, se ubican en el primer período del pensamiento de Heidegger, y 2) que la "amenaza" de la técnica, que se extrae del análisis de entes técnicos (tales como la planta hidroeléctrica) tiene su génesis en la incorporación del útil artesanal a un sistema individualizado de útiles que exhiben una cierta secuencialidad iterativa ${ }^{33}$, es decir, desde el punto de vista del artefacto, el peligro comienza cuando el útil es integrado como elemento de una máquina (individuo técnico), a su vez, el peligro crece exponencialmente, hasta llegar a su máxima expresión cuando la máquina se inscribe en el interior de una red plurisistemática donde se entrecruzan actores sociales y materiales de muy diversas índoles (desde el sistema del tendido eléctrico hasta el sistema de traslado de materiales, hasta el colectivo gremial que agrupa a los distribuidores). Dicho en otras palabras, el peligro alcanza "masa crítica" cuando el antes inocuo útil artesanal se amplifica lo suficiente para llegar a constituir un nodo en un conjunto técnico ${ }^{34}$, este conjunto puede interpretarse como una versión expandida del mundo circundante del primer Heidegger ${ }^{35}$.

Este último intento de contrastación también ha fracasado, en esta oportunidad, porque los intereses filosóficos del primer Heidegger (período en el que se estudia el martillo) no coinciden con los del segundo (período en que se estudia la "esencia de la técnica"). Ciertamente es posible que la simple sumatoria de pequeños martillos inofensivos en una máquina la vuelvan escenario de la Gestell, pero no por el funcionamiento mecánico de la misma ni por la integración y movimiento de sus partes, si no por la instalación de la máquina de escribir en un contexto epocal y cultural, esto es, por su aparición dentro de la historia del olvido del ser. La máquina de escribir no es aquí precisamente un objeto técnico concreto y operativo, sino más bien un índice o arquetipo del modo calculador de relación con el mundo, que posterga a la palabra en tanto "casa del ser".

\section{Conclusión: los límites del ejemplo}

\footnotetext{
${ }^{33} \mathrm{G}$. Simondon, El modo de existencia de los objetos técnicos, Buenos Aires, Prometeo, 2007, p. 162.

${ }^{34}$ G. Simondon, Ibid.., p.83.

${ }^{35}$ Cf. L. Mascaró, "Claves para repensar el mundo circundante" en Tópicos. Revista de Filosofía de Santa Fe, Nún. 38, 2019, pp. 115-143. 
Para finalizar, expongamos brevemente, pero de manera manifiesta la cuestión que se encontraba en el trasfondo de los intentos de contrastación que realizamos en este artículo: ¿cómo deben ser leídos los ejemplos en Heidegger, y qué relevancia tienen dichos ejemplos, y sus análisis correspondientes, en el marco del análisis filosófico de objetos técnicos? Para responder a esta pregunta, atendamos a la información que nos han aportado nuestros intentos fallidos. Si las afirmaciones heideggerianas acerca de un objeto técnico como la máquina de escribir escapan a la indicación de su imprecisión histórica, eluden el señalamiento de su imprecisión paleontológica, esquivan a la mostración de su imprecisión fenomenológica (y postfenomenológica), e incluso evitan la inconsistencia que se observa con otras afirmaciones del propio Heidegger, entonces su estudio de casos se nos muestra como inmune a la contrastación con información científica y experiencial. Es imposible indicar una contradicción empírica en la filosofía de Heidegger, puesto que, como hemos visto una y otra vez, su interés se mueve en otras direcciones. ¿Cuál es entonces el valor de un ejemplo en la filosofía de este autor? El pensador nunca ofrece una indicación explícita sobre cómo deben ser leídos sus ejemplos.

Varios autores se han dedicado a la investigación del valor de los ejemplos en la filosofía en general, y en el pensamiento de Heidegger, en particular. John Dewey, en su obra Art as Experience, realiza una presentación del ejemplo como una "experiencia simulada"36. Para John D. Lyons, los ejemplos son capaces de otorgar "riqueza epistémica" a la certeza sensible, cuyo valor suele ser relegado en los tratados filosóficos. Según el autor, el ejemplo permite alcanzar un suelo común de creencia en un terreno que escritores y lectores reconocerán como real. Los ejemplos traen consigo, de manera implícita, las reglas según las cuales algo puede ser visto como creíble ${ }^{37}$. Según N. Goodman, la ejemplificación consiste en una transformación de un particular en algo ejemplar. Esta transformación se lleva a cabo por vía de la reducción de el evento o cosa a una serie de características como representantes del "algo" concreto. Las condiciones mínimas para la producción de la ejemplaridad consisten, en la posesión material y la formación de una referencia demostrativa ${ }^{38}$. Una postura tal vez más compatible con el modo de empleo de los ejemplos en Heidegger la encontramos en G. Agamben, para quien el ejemplo produce lo singular mediante la analogía. Según su postura, en un ejemplo sería imposible dividir el carácter de paradigma con valor universal y su carácter singular, por tanto, el ejemplo funcionaría, precisamente como aquel recurso capaz de hacer presente la relación entre dos singulares, como un campo de fuerzas opuestas, donde la tensión no se resuelve, sino que, precisamente, se vuelve manifiesta. El ejemplo sería a la vez, lo paradigmático y lo singular, o más precisamente, la tensión analógica entre estos dos caracteres ${ }^{39}$.

Una autora que se ha dedicado puntualmente al análisis de los ejemplos en Heidegger es M. Schaub. La filósofa desarrolla una serie de valiosas nociones: en primer lugar, presenta al ejemplo como el lugar privilegiado para la producción de lo singular. Para ella, el ejemplo funciona como una miniaturización, esto es, una totalidad contenida en una parte; al mismo tiempo, esta totalidad reducida presenta una estructura narrativa. Adicionalmente, el ejemplo conserva una relación con lo sensible. Para la autora, el ejemplo constituye "una astilla de certeza sensible en el texto filosófico" 40 . Schaub presenta una interesante crítica al uso heideggeriano de los ejemplos ${ }^{41}$, según la

${ }^{36}$ J. Dewey, Art as Experience. New York, USA: Wideview/Perigee, 1985, pp. 273 y 178.

${ }^{37}$ J. D. Lyons, Exemplum, The rethoric of example in early modern France and Italy, New Jersey. Princeton University Press, 1989, p.10

${ }^{38}$ N. Goodman, Languages of Art. An Approach to a Theory of Symbols. Indianapolis/New York/Kansas: Bobbs Merrill Piblishers, 1968 , p. 52 y ss.

${ }^{39}$ G. Agamben, Signatura rerum. Buenos Aires. Adriana Hidalgo [Tr. Esp. Flavia Costa y Mercedes Ruvituso], 2009, p. 27-28.

${ }^{40}$ M. Schaub, Das Singulare und das Exemplarische: Zur Logik und Praxis der Beispiele in Philosophie und Asthetik. Zürich, Diaphanes, 2010, p.232.

${ }^{41}$ La autora se concentra especialmente en el análisis del texto de Heidegger "el origen de la obra de arte". 
filósofa, Heidegger hace uso en sus ejemplos de dos movimientos contrapuestos; por un lado, el control total del caso concreto, lo cual conlleva la eliminación de su singularidad, y por el otro un "dejar hacer", que consiste en la transformación de un ejemplo controlado, y propenso de ser sometido a crítica en lo que ella llama "un paradigma ingobernable" que no puede ser criticado ${ }^{42}$. El proceso por el cual el singular es convertido en un paradigma inmune a la crítica es denominado "esencialización". Como consecuencia de su descripción del uso heideggeriano de ejemplos, la autora concluye que, para el autor alemán, "Lo singular tiene un lugar poco relevante. $Y$ desde un punto de vista teórico carece en absoluto de toda ponderación ${ }^{n 43}$.

Ahora bien, también es posible encontrar autores que argumentan que el uso metodológico del ejemplo en Heidegger persigue, por medio de sus aparentes controles, sesgos o limitaciones, un objetivo puntual y fundamental: la mostración de la diferencia ontológica. Entre estos autores encontramos a Bertorello ${ }^{44}$, quien regresa a la concepción de Agamben para indicar que el ejemplo heideggeriano, efectivamente cumple la función de indicar una tensión entre dos singulares, solo que en este caso, los elementos no serían una función paradigmática y un caso sensible particular, sino la tensión entre ser (como Ereignis) y ente, es decir, la diferencia ontológica.

Más allá de las diversas perspectivas que revisamos muy sucintamente ${ }^{45}$, parecieran percibirse dos situaciones fundamentales en lo que a nuestra temática refiere: 1) Según diversas perspectivas filosóficas, los ejemplos en general, y los empleados en textos filosóficos en particular, parecieran tener una referencia esencial a la experiencia, a lo singular, y últimamente, a lo sensible, 2) los ejemplos en la filosofía de Heidegger no parecen plegarse al esquema recién mencionado, ya que en ningún caso el ejemplo heideggeriano queda asociado de manera prioritaria a los datos sensibles, y tampoco parecen cumplir con una función inductiva o didáctica.

Lo que se esconde detrás de la advertencia de Heidegger es la limitación empírica de su filosofía, el verdadero rango del ejemplo. Como indicaba Schaub, el ejemplo heideggeriano se expande hasta llegar a la dimensión de un paradigma ingobernable. Aún si adscribimos a la defensa que indica que los ejemplos en Heidegger no son imprecisos, ni desdibujan lo particular, sino que buscan un efecto intencionado, deberemos aceptar que lo particular en tanto ente y Ereignis (los dos términos tensionados en la diferencia ontológica), no constituyen un algo particular en sentido empírico, sensible, o últimamente, contrastable con la experiencia, y propenso de ser sometido a prueba, crítica y revisión.

Ahora bien, esa inmunidad delinea ante nosotros un tipo muy especial de filosofía, y trae muy importantes consecuencias en lo que a nosotros nos ocupa: la aplicabilidad de los dichos de Heidegger al estudio de las tecnologías concretas y empíricamente situadas con las que nos relacionamos cotidianamente. Este es el límite de la filosofía de Heidegger si se desea aplicarla a la filosofía de la técnica en tanto estudio filosófico de elementos técnicos empíricos. Si la máquina de escribir, la escritura, (o en otras obras, el puente, la jarra, la copa sacrificial, el templo) no son ejemplos empíricos ni estudios de artefactos concretos, sino "índices", "arquetipos", "singulares esencializados", o "campos de fuerzas", utilizados para señalar un fenómeno ontológico posibilitante, y en general, el trasfondo del mundo como espacio de acontecimiento de la verdad del ser, entonces debemos estar prevenidos de que poco o nada se podrá obtener de la aplicación de los dichos de Heidegger al estudio de los artefactos concretos y operativos que pueblan el mundo.

Heidegger (específicamente, después de los años '30) no es un investigador de la tecnología, sino de la metafísica de la tecnología. Muy rápidamente desestima el necesario análisis de artefactos concretos en favor de la más abstracta (pero por lo

${ }^{42}$ M. Schaub, Ibid., p.277

${ }^{43}$ Cf. Schaub, Ibid。, pp 332 y ss.

${ }^{44} \mathrm{~A}$. Bertorello, "La fuerza argumentativa del ejemplo del cuadro de van Gogh en Der Ursprung des Kunstwerkes", En Anuario Heidegger. Número 1, Ed. Herder.-En prensa-, 2020.

${ }_{45}^{4}$ Revisión que de ningún modo pretende abarcar la variedad de posturas, ni agotar la temática en cuestión. 
mismo, más precisa e incuestionable) "esencia de la técnica"; y, dado que, como él mismo indica, esta esencia "no es nada técnico" ", y que el ciclotrón, la turbina de vapor o la emisora de radio no son una estructura de emplazamiento, y que la Gestell (esencia de la técnica) no mienta ningún aparato ni tipo de maquinaria ${ }^{47}$, nuestro autor se permite el abandono de la instancia "experimental" de la filosofía $a^{48}$. Heidegger se ubica demasiado lejos de los fenómenos técnicos. En consecuencia, los resultados y las conquistas de sus investigaciones posteriores a los '30 deberían quedar restringidas al ámbito que él mismo delimitó para ellos: el ámbito ontológico y trascendental.

\section{Referências}

AGAMBEN, G. (2009) Signatura rerum. Buenos Aires. Adriana Hidalgo [Tr. Esp. Flavia Costa y Mercedes Ruvituso].

ADKINS, L. (2003) Empires of the plain, New York, St. Martin's press.

ARCADI, A.C. (2000), "Vocal responsiveness in male wild chimpanzees: implications for the evolution of language". En Journal of human evolution, 39 2, 205-23.

AUBERT, M., SETIAWAN, P., OKTAVINA, A.A. et al. (2018) "Palaeolithic cave art in Borneo". En Nature no 564 , p. 254-257

BERTORELLO (2006) "La polémica en tomo a la estética ontológica de Heidegger: Schapiro, Schaeffer y Derrida", en Contrastes, revista internacional de filosofía, Ed. Universidad de Málaga: España

BERTORELLO (2020) "La fuerza argumentativa del ejemplo del cuadro de van Gogh en Der Ursprung des Kunstwerkes", En Anuario Heidegger. No 1, Ed. Herder.-En prensa-

BOORSTIN, D. (1986) Los Descubridores, trad. de Susana Lijtmaer.Ed. Grijalbo: Barcelona

DERRIDA, J. (1996) "El tratamiento del texto", Entrevista con Béatrice y Louis Seguin, La quinzaine littéraire, 698, pp. 4-7, traducción de R. Tbáñez y M. J. Pozo en No escribo sin Iuz artificial, cuatro ediciones, Valladolid, 1999.

ENGESSER, S. et Al. (2015) Experimental Evidence for Phonemic Contrasts in a Nonhuman Vocal System en Plos Biology, Princeton: Estados Unidos

DEWEY, J. (1985). Art as Experience. New York, USA: Wideview/Perigee

FOUCAULT, M. (2008) Las palabras y las cosas, Siglo XXI, Buenos Aires, Argentina.

GOODMAN, N. (1968) Languages of Art. An Approach to a Theory of Symbols. Indianapolis/New York/Kansas: Bobbs Merrill Piblishers

HEIDEGGER (1994) "La pregunta por la técnica", en Conferencias y artículos, ed. del serbal:Barcelona

HEIDEGGER (2005) Parmenides. AKAL, Parmenides. Traducción de Carlos Másmela. Madrid: Akal

HEIDEGGER (2006) Ser y Tiempo, Trotta, Madrid, 2006

IHDE. D. (2010) Heidegger's technologies: Postphenomenological perspectives. Fordham University Press: New York

IHDE. D. (1979) Technics and praxis, Ed. Reidel Publishing Co.,Dordrecht: Holland.

${ }^{46}$ M. Heidegger, "La pregunta por la técnica", en Conferencias y artículos, Barcelona, Ed. del Serbal, 1994, p.9.

${ }^{47}$ M. Heidegger, Ibid., p. 13

${ }^{48}$ D. Ihde, Experimental phenomenology, State University of New York Press, Albany, 2012, p.5. 
IHDE. D. (2012) Experimental phenomenology, State University of New York Press, Albany.

LYONS, J.D. (1989) Exemplum, The rethoric of example in early modern France and Italy, New Jersey: Princeton University Press.

MASCARÓ, L. (2019) "Claves para repensar el mundo circundante" en Tópicos. Revista de Filosofía de Santa Fe, Núm. 38, pp 115-143

MERLEAU-PONTY, M. (1994) Fenomenología de la percepción, Ed. Planeta Agostini, Barcelona: España.

MIYAGAWA, S., BERWICK, R. Co, \& OKANOYA, K. (2013). The emergence of hierarchical structure in human language. Frontiers in psychology, 4, 71

RICOEUR (2006) "la función lhermenéutica del distanciamiento", en Del texto a la acción, Fondo de Cultura Económica: Buenos Aires

SCHAPIRO, M. (1999): "La naturaleza muerta como objeto personal: unas notas sobre Heidegger y Van Gogh", en Schapiro M, Estilo, artista y sociedad. Teoría y filosofía del arte. Madrid: Tecnos.

SCHAUB, M. (2010) Das Singulare und das Exemplarische: Zur Logik und Praxis der Beispiele in Philosophie und Ästhetik. Zürich, Diaphanes

SCHMIDT, H-M.; LANZ, U. (2003) Surgical Anatomy of the Hand. Ed. Thieme.

SIMONDON (2007) El modo de existencia de los objetos técnicos, Prometeo: Buenos Aires

Doutor em Filosofia Professor CONICET/UBA

E-mail: Icnmascaro@hotmaill.com 